\title{
DỨcin
}

Technological University Dublin

ARROW@TU Dublin

2010-01-01

\section{A Liquid Crystal Coated Tapered Photonic Crystal Fiber Interferometer}

\author{
Ginu Rajan \\ Technological University Dublin, ginu.rajan@tudublin.ie \\ Sunish Mathews \\ Technological University Dublin \\ Gerald Farrell \\ Technological University Dublin, gerald.farrell@tudublin.ie
}

See next page for additional authors

Follow this and additional works at: https://arrow.tudublin.ie/engscheceart

Part of the Electrical and Computer Engineering Commons

\section{Recommended Citation}

Rajan, G. et al. (2010) A Liquid Crystal Coated Tapered Photonic Crystal Fiber Interferometer. Journal of Optics, Vol. 13, December 2010, 7p. doi:10.1088/2040-8978/13/1/015403

This Article is brought to you for free and open access by the School of Electrical and Electronic Engineering at ARROW@TU Dublin. It has been accepted for inclusion in Articles by an authorized administrator of ARROW@TU Dublin. For more information, please contact arrow.admin@tudublin.ie, aisling.coyne@tudublin.ie, gerard.connolly@tudublin.ie.

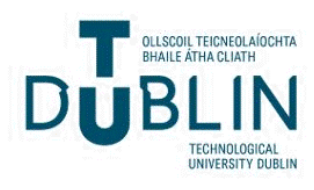




\section{Authors}

Ginu Rajan, Sunish Mathews, Gerald Farrell, and Yuliya Semenova 


\title{
A Liquid Crystal Coated Tapered Photonic Crystal Fiber Interferometer
}

\author{
Ginu Rajan*, Sunish Mathews, Gerald Farrell and Yuliya Semenova \\ Photonics Research Centre, School of Electronic and Communications Engineering, Dublin Institute \\ of Technology, Kevin Street, Dublin 8, Ireland \\ *E-mail: ginu.rajan@dit.ie
}

\begin{abstract}
An experimental investigation on liquid crystal coated tapered photonic crystal fiber (PCF) interferometer is presented in this paper. The interferometer is fabricated by tapering a small section of a PCF by collapsing the air holes and thinning down the air hole collapsed region to a micron size. Several interferometers are fabricated from different types of photonic crystal fibers and the temperature dependence studies are carried out to select an intrinsically temperature insensitive interferometer that can be used with liquid crystal (LC) materials. The properties of the LC materials to be used with the tapered PCF interferometers are also discussed. Temperature tuning of the spectral response of the tapered interferometers with different waist diameters coated with high index and low index LC materials are carried out and the characteristics are presented.
\end{abstract}

Pacs No: 42.81

Keywords: Tapered PCF interferometer, liquid crystal

Submitted to: Journal of Optics A: Pure and Applied Physics

\section{Introduction}

Photonic crystal fiber (PCF) interferometers can be fabricated in a number of ways and their properties and applications have been studied by many authors [1-5]. Different types of PCF interferometers include fiber loop mirror interferometers, micro-hole collapsed interferometers, tapered interferometers and hollow core Fabry-Perot interferometers. These interferometers are used in many applications such as strain sensing, refractive index sensing, bio- sensing, gas sensing and temperature sensing [1-9]. The properties of a PCF interferometer and its sensitivity to different measurands are determined by the type of interferometer, its geometry and by the type of the PCF used. One of the most compact types of PCF interferometers is the tapered interferometer. A PCF taper shows similar characteristics to those observed in abruptly tapered single-mode fibers [10]. However a tapered PCF interferometer shows much higher sensitivity than tapered interferometers based on a singlemode fiber. Also a singlemode fiber taper exhibits a temperature dependence at least 2-3 times higher than that of PCF tapers due to the presence of dopants in the fiber [1112]. PCF on the other hand, due to its pure silica nature, exhibits very low inherent temperature dependency. Hence the possibility to control the properties of such a tapered PCF interferometer externally without changing its structure could add a further degree of control in many applications. One possible method to realize such control is by coating the interferometer with a layer of a liquid crystal material whose refractive index is susceptible to changes in temperature or the magnitude of an external electric field. In the investigation presented in this paper we have studied the effects of thermally controlled changes in the 
characteristics of several tapered PCF interferometers coated with nematic liquid crystal materials.

Nematic liquid crystals are highly anisotropic materials whose refractive indices are dependent on temperature or external fields [13-14]. When a tapered PCF interferometer is coated with a nematic liquid crystal material, the propagation properties of the interferometer change as a result of changes in the refractive indices of the LC with temperature resulting in a temperature induced spectral shift of the interference pattern. To analyze the spectral behaviour of an LC coated tapered interferometer with change in temperature an experimental characterization study is carried out and is presented in this paper. In section 2 the fabrication of the tapered PCF interferometers is discussed. The temperature dependence studies of the interferometers made from different types of PCF are also presented in this section. The properties of the LC materials to be used with the tapered PCF interferometers are discussed in section 3. An experimental demonstration of temperature tuning of the spectral response of a LC coated tapered PCF interferometer is presented in section 4. Experimental results for fiber tapers coated with low index and high index LC materials are also presented in this section. Interferometers with different taper waist diameters are fabricated and characterized and it is found that the temperature sensitivity depends on the waist diameter of the tapered fiber interferometer.

\section{Tapered PCF interferometers}

\subsection{Fabrication of the tapered PCF interferometers}

To make a tapered PCF interferometer sensitive to the external environment, the cladding modes in the fiber should be excited [1]. Therefore a PCF with large number of rings and large mode field diameter should be considered for the interferometer fabrication. Three different types of photonic crystal fibers are used in this experimental analysis. We have considered LMA-8, LMA-10 and polarization maintaining PM1550-01 photonic crystal fibers. LMA-8 has 7 rings of holes and a core diameter of $8.5 \mu \mathrm{m}$, with a mode field diameter of $6 \mu \mathrm{m}$. The LMA-10 fiber has a core diameter of $10 \mu \mathrm{m}$ with the 7 rings of holes and a mode field diameter of $7.5 \mu \mathrm{m}$. The cladding diameter of both LMA fibers was $125 \mu \mathrm{m}$. PM1550-01 is a highly birefringent fiber with an asymmetric core and with 5 rings of holes surrounding the core region. The mode field diameters are 3.6 (long axis) $\mu \mathrm{m}$ and 3.1(short axis) $\mu \mathrm{m}$ and the spacing between adjacent holes is $4.4 \mu \mathrm{m}$. The diameter of the holey region is $40 \mu \mathrm{m}$ and the total diameter of the fiber is $125 \mu \mathrm{m}$.

Initially for the fabrication of the tapered interferometer a small section (approx $3 \mathrm{~cm}$ ) of the PCF is spliced on to a standard singlemode fiber pigtail. Using a carefully controlled fusion splicing process ensured that no interference patterns arose due to hole collapse in the PCF during splicing [15]. The splice losses at both ends were approximately $3 \mathrm{~dB}$. The central region of the PCF was then collapsed and thinned down to a micron size to form the tapered region. This is carried out by using a standard splicing machine and while applying the electric arc the fiber is pulled from both sides uniformly. The tapered region formed in the PCF is similar to an unclad multimode optical fiber. As a consequence of this, the fundamental mode in the PCF in the region where the holes are open is coupled to the modes of the solid fiber in the tapered region and as a result of this mode coupling, beating between the multiple modes takes place and the transmission spectrum shows an oscillatory behaviour [9]. By controlling the taper waist diameter and length the performance of the interferometer can be adjusted. An image of one of the fabricated PCF taper is shown in Fig. 1. It should be noted that during the fabrication the arc is applied to a point region while the fiber is pulled from both sides. As a result it is difficult to achieve a precise control over the taper diameter and hence the taper length will vary for different samples; the tapers with lower waist diameters will have longer lengths. Both the taper diameter and length influence the period of the interference spectrum. It was observed that the lower the waist diameter and the longer the 


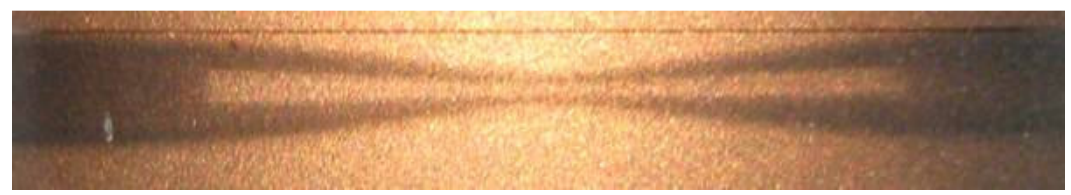

Figure 1. An image of one of the fabricated PCF tapers.

taper length the smaller the period of interference. For comparison studies of the PCF interferometers made from different fiber tapers we chose several tapers with approximately the same length and waist diameter from a number of fabricated samples.

Interference patterns in the transmission spectrum occur only when the tapered region diameter is less than $40 \mu \mathrm{m}$, as only below this thickness do the holes collapse and a multimode region is formed in the central region of the PCF $[9,16]$, which leads to the interference between the modes in the fiber. The fabricated PCF tapers had a length of approximately $0.3 \mathrm{~mm}$ with a waist thickness of approximately $19 \mu \mathrm{m}$ for all the three fiber types. The measured spectra of all the tapered PCF interferometers are shown in Fig 2. The spectra are measured in a wavelength region between $1510 \mathrm{~nm}$ to $1580 \mathrm{~nm}$. From the figure multiple peaks can be seen for all the three interferometers. More closely spaced peaks can be obtained by reducing the waist diameter of the tapered region which can be controlled by adjusting the pulling forces from both sides during splicing.

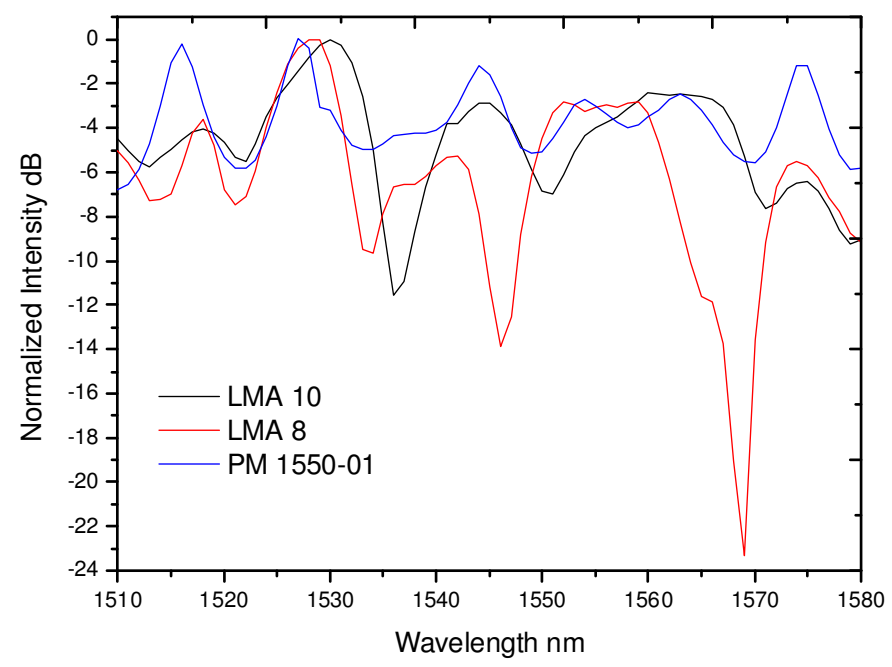

Figure 2. Measured spectra of the fabricated tapered PCF interferometers using different fiber types

\subsection{Temperature dependence of tapered PCF interferometers}

In order to study the influence of temperature on an LC covered tapered interferometer which arises from the temperature dependence of the LC refractive indices, it is necessary to reduce or eliminate, in as far as it is practical, the intrinsic temperature dependence of the interferometer in the absence of the LC layer. One known factor influencing the temperature dependence of an uncoated interferometer is the type of PCF used. Several authors have previously reported temperature sensitive and insensitive tapered PCFs, and we believe that the different temperature dependencies are a result of the differences in geometry of the PCFs $[1,9]$. However in general for any PCF taper, temperature dependency is very low compared to singlemode fiber tapers $[11,12]$. The influence of temperature on the parameters of the interferometers described above was investigated. To evaluate the temperature dependence, 
each of the fabricated interferometers was attached in turn to a Peltier cooler driven by a temperature controller. The temperature was varied from $20{ }^{\circ} \mathrm{C}$ to $60{ }^{\circ} \mathrm{C}$ in intervals of $10{ }^{\circ} \mathrm{C}$ and the wavelength shifts were measured from the spectral responses and are shown in Fig. 3 for the three interferometers. From Fig. 2 it can be seen that the interferometers made from LMA-8 and PM-1550-01 fibers are temperature dependent, while for the interferometer made from LMA-10 fiber the temperature dependent wavelength shift is negligible. On this basis the most suitable interferometers to be used with the LC material are those made from the LMA-10 fiber.

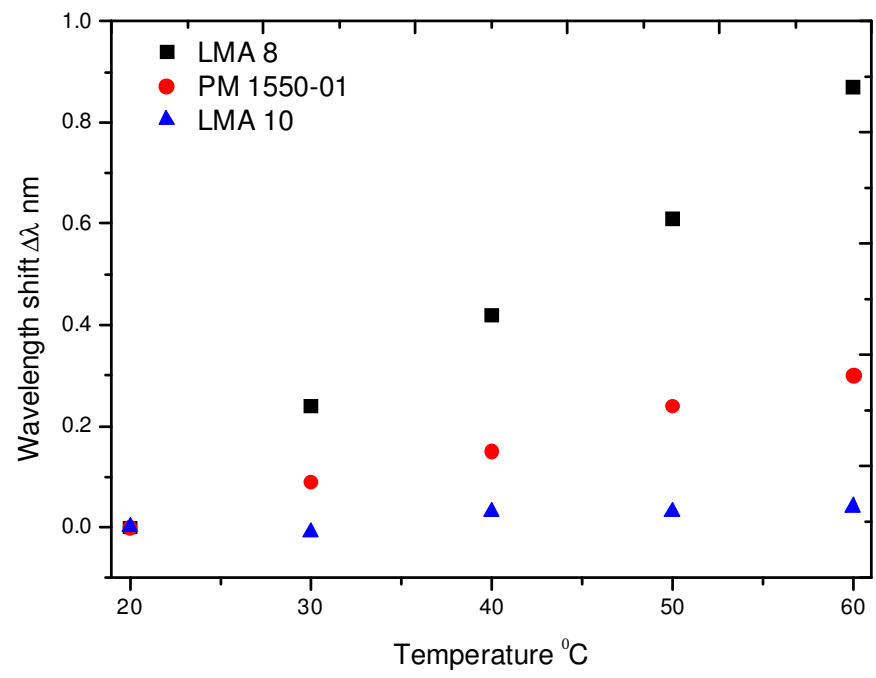

Figure 3. Temperature dependent wavelength shift for the fabricated interferometers

\subsection{LMA-10 based tapered interferometers}

In the case of interferometers fabricated from LMA-10 fiber a wavelength shift of the spectrum can be caused either by application of a strain or by the influence of a change in the surrounding refractive index. In the latter case the external medium plays the role of cladding and the tapered solid section plays the role of core. Therefore, an external medium surrounding the region with collapsed air-holes will attenuate or absorb the evanescent fields of the different propagating modes. As a consequence, the interference pattern of the taper will also be modified. If the refractive index of the external medium is lower than that of silica, then the external medium interacts with the cladding modes, shifting the wavelength and allowing such an interferometer to act as a refractive index sensor. If the external medium has a refractive index higher than that of silica then the cladding modes will refract into the medium and will radiate away and hence the extinction ratio of the interference spectrum will be reduced and no shift in the wavelength will take place with a change in the refractive index of the external medium.

To study the influence of the refractive index of a LC material on the tapered PCF interferometer, four interferometers were fabricated with different waist thickness, using LMA-10 PCF. The fabricated LMA-10 tapers have waist diameters of $13 \mu \mathrm{m}, 19 \mu \mathrm{m}, 22 \mu \mathrm{m}$ and $35 \mu \mathrm{m}$ and measured 1engths of $0.41 \mathrm{~mm}, 0.3 \mathrm{~mm}, 0.27 \mathrm{~mm}$ and $0.21 \mathrm{~mm}$ respectively. The measured spectra of the interferometers with different waist diameters are shown in Fig 4. It can be seen that as the taper diameter decreases, the interferometer's spectrum exhibits more peaks and the peaks become sharper. This is because the beat length of the modes in the solid taper decreases with the decrease in the taper diameter since the separation between the propagation constants of the modes increases [9]. 


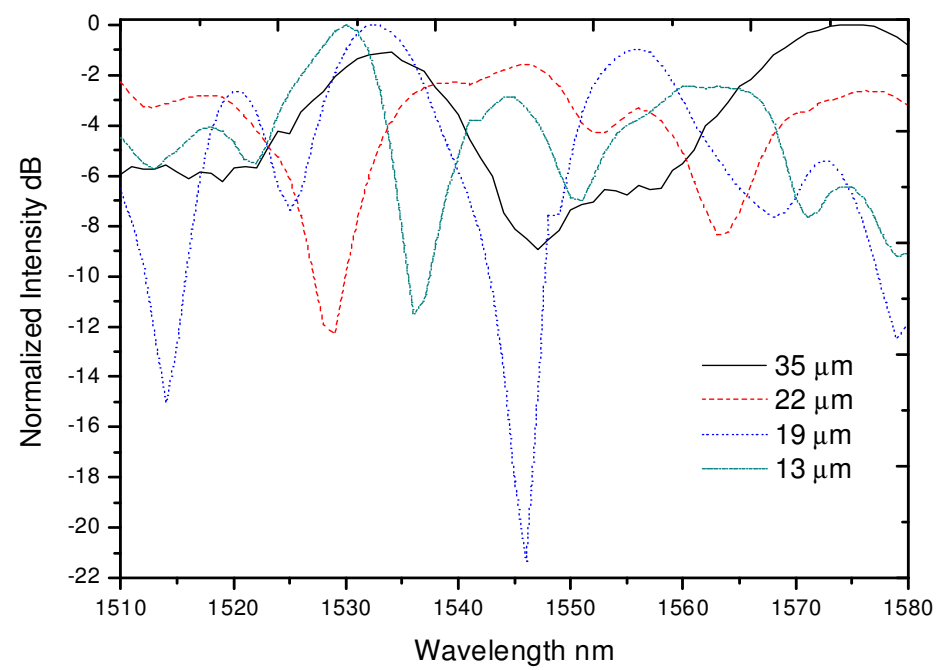

Figure 4. Spectral response of the LMA-10 PCF interferometers with different waist diameter

\section{Liquid crystal materials for tapered PCF interferometers}

In the selection of an appropriate nematic LC material for an LC coated interferometer the ordinary $\left(\mathrm{n}_{\mathrm{o}}\right)$ and extra-ordinary $\left(\mathrm{n}_{\mathrm{e}}\right)$ refractive indices of the LC material and their temperature dependence play a vital role. LC refractive indices are determined by the molecular structure of the LC material, the operating wavelength and the temperature [13]. With an increase of temperature, variations in $n_{e}$ and $n_{o}$ occur, which in turn leads to a decrease in the average refractive index of the liquid crystal material, given by the equation [14]:

$$
\langle n\rangle=\frac{n_{e}+2 n_{o}}{3}
$$

By selecting a liquid crystal whose average refractive index is below the refractive index of silica and by changing the temperature dependent effective refractive index, the propagation properties of the fiber interferometer can be altered and hence control over the interference pattern can be realized. However the value of the wavelength shift depends on the birefringence of the LC material which decreases as the temperature increases and at the temperature of transition to the isotropic phase (clearing point) the birefringence reduces to zero.

It should be noted that LC materials with refractive indices (both $n_{e}$ and $n_{0}$ ) lower than that of silica are not commercially available. In this study we used a prototype mixture cat. no. 1550 manufactured by the Military University of Technology in Warsaw as a low index LC [17]. The $\mathrm{n}_{\mathrm{o}}$ and $\mathrm{n}_{\mathrm{e}}$ of 1550 is 1.46 and 1.52 respectively at $589 \mathrm{~nm}$. As a high refractive index LC material commercially available 5CB from Merck Ltd. was used in this study. The $n_{o}$ and $n_{e}$ of $5 \mathrm{CB}$ are 1.52 and 1.71 respectively at $589 \mathrm{~nm}$. In the case of $1550 \mathrm{LC}$, the average refractive index is close or below to the refractive index of silica, but the refractive index of $5 \mathrm{CB}$ it is higher than that of the silica and hence these materials were chosen to provide an insight into the effect of the relationship between the refractive index of silica and that of the LC material used.

\section{Liquid crystal coated PCF interferometers}

\subsection{Response of the interferometer covered with a low index LC layer}

The four fabricated tapered PCF interferometers with different waist diameters based on LMA-10 fiber were coated with a layer of a low index 1550 liquid crystal material. Each 
interferometer was sandwiched between two glass plates for mechanical stability. In this arrangement the LC material can be applied to easily obtain a uniformly coated layer around the tapered region of the interferometer. The orientation of the LC molecules on the surface of the tapered fiber is not controlled by any pre-alignment techniques. The spectral responses of the LC coated interferometers are obtained at room temperature $\left(20{ }^{\circ} \mathrm{C}\right)$. The measured spectra of all the interferometers are shown in Fig 5. It is observed from the figures that the number of peaks remains the same when compared to the uncoated interferometers but as a result of the presence of the LC material, the responses become smoother and also there is a slight decrease in the extinction ratio of the interferometer. This is possibly due to the fact that the refractive index contrast between the fiber and air is higher than the refractive index contrast between the fiber and the LC material.

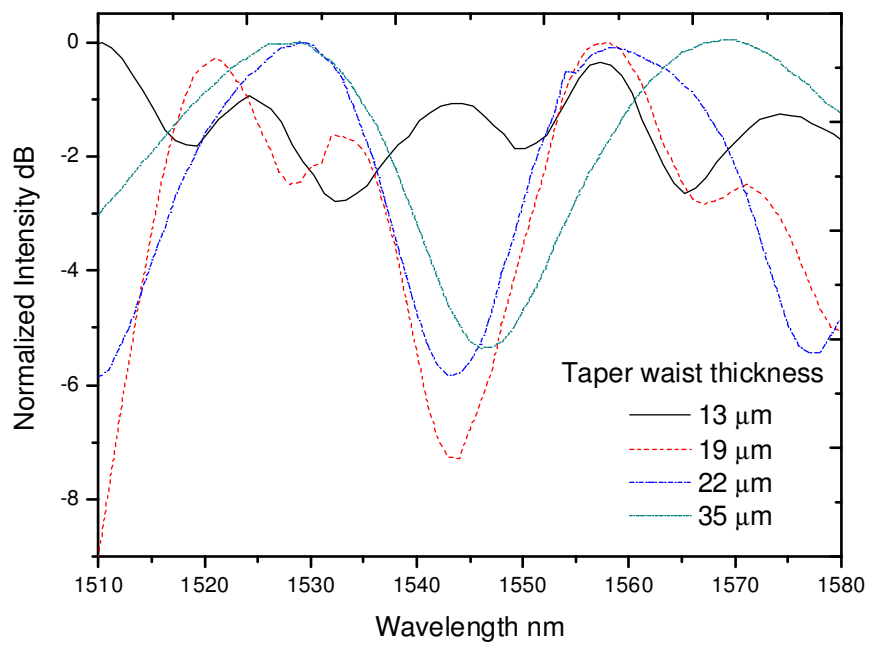

Figure 5. Spectral response of the LC coated PCF interferometers with different waist diameter

To study the temperature tuning properties of the LC coated interferometer, the structure is placed on the top of a Peltier cooler and the temperature is varied from $20{ }^{\circ} \mathrm{C}$ to $60{ }^{\circ} \mathrm{C}$ at $10{ }^{\circ} \mathrm{C}$ intervals. The Peltier cooler is controlled using a temperature controller. The temperature range was limited by the Peltier cooler but the upper limit is acceptable since the clearing point temperature of the $1550 \mathrm{LC}$ material is $78{ }^{\circ} \mathrm{C}$ and the refractive index variation reduces when the temperature is close to the clearing point. The experimental setup used for the temperature study is shown in Fig 6.

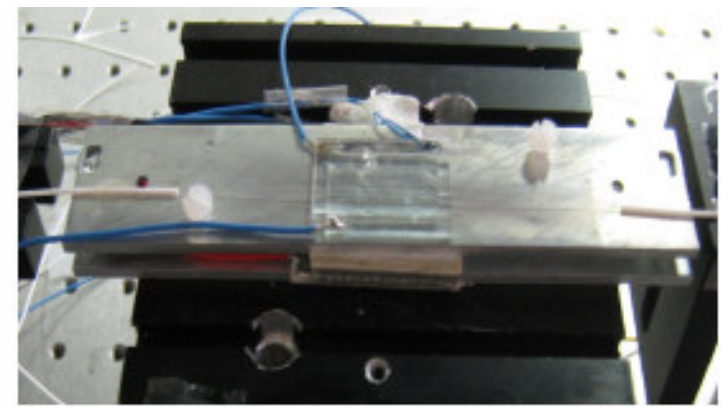

Figure 6. Experimental arrangement to study the temperature tuning of the LC coated tapered PCF interferometer

The measured spectral responses for all four LC coated interferometers with different waist diameters at $20{ }^{\circ} \mathrm{C}$ and $60{ }^{\circ} \mathrm{C}$ are shown in Fig 7(a-d). From the figure it is clear that all the spectra show a blue shift with an increase in temperature. This is because as the temperature 


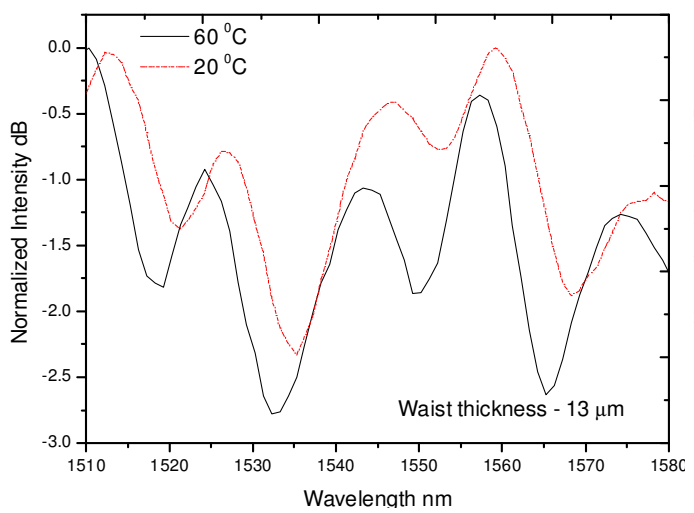

(a)

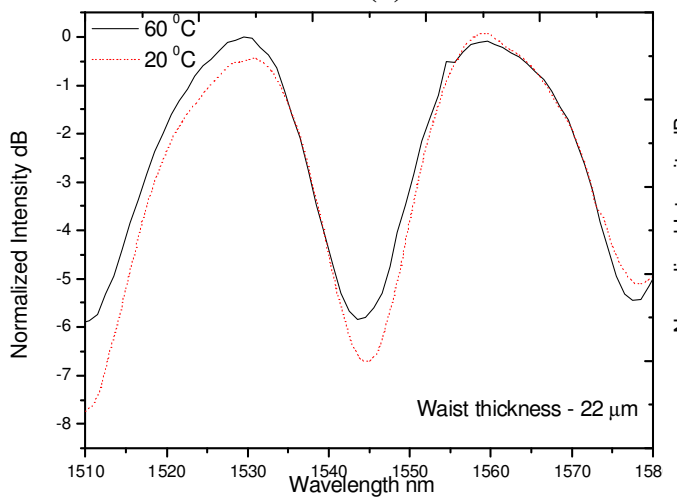

(c)

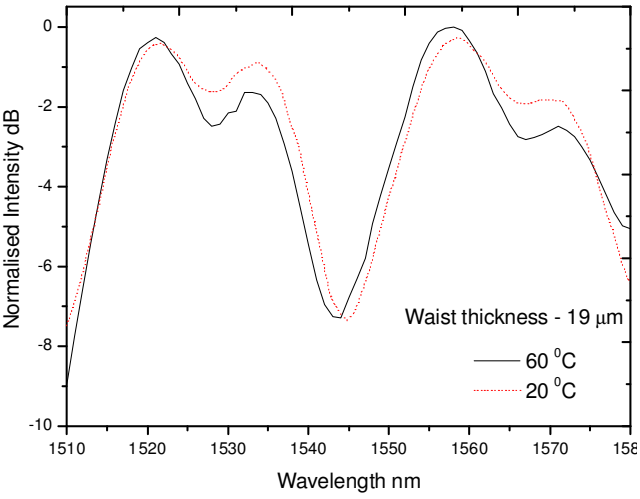

(b)

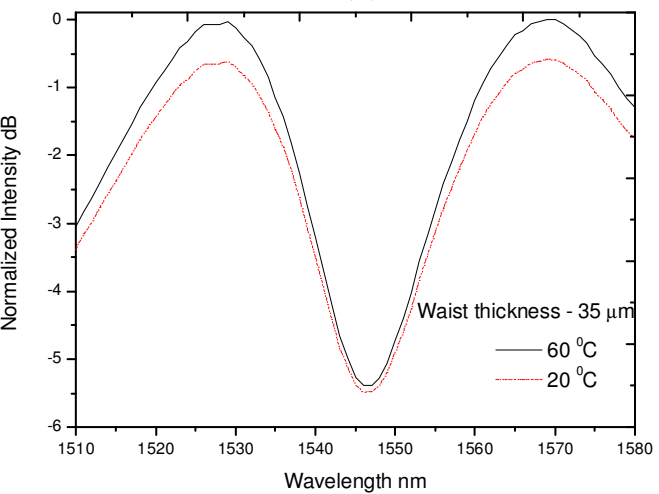

(d)

Figure 7. Observed wavelength shift for LC coated PCF interferometers with different waist diameters

increases the average refractive index of the LC material decreases [9, 17]. From the figure it can be seen that the interferometer with a waist diameter of $13 \mu \mathrm{m}$ shows the largest shift and the value of the wavelength shift decreases as the taper waist diameter increases. The wavelength shift measured for all the interferometers and also the wavelength sensitivity of the interferometers are shown in Fig 8(a) and Fig 8(b) respectively. For the interferometer with a waist thickness of $35 \mu \mathrm{m}$ no significant wavelength shift was observed. This is because as the waist diameter increases the modes participating in the interference are low order modes and the higher order modes which interact strongly with the surrounding LC layer are

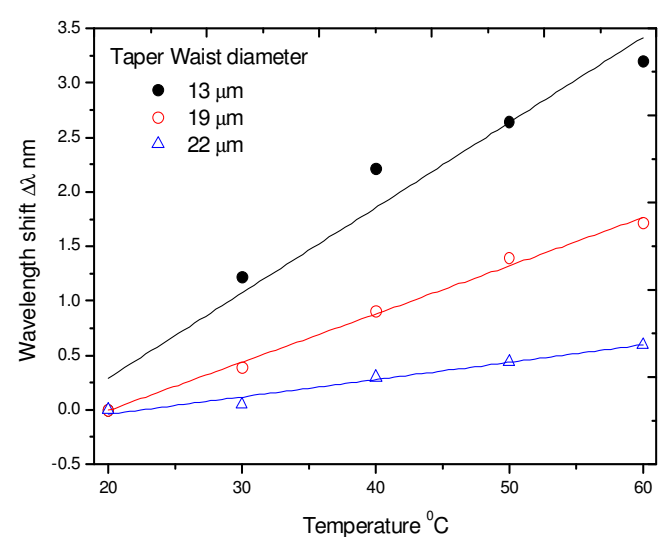

(a)

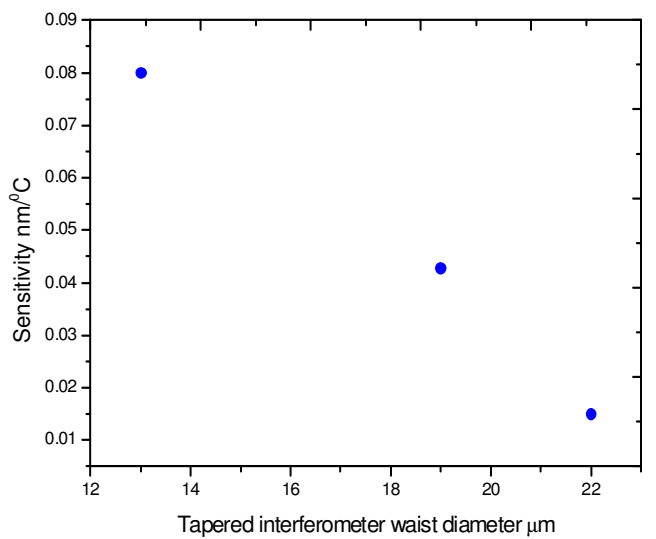

(b)

Figure8. (a) Measured wavelength shift for the interferometers with different waist diameters (b) Sensitivity of the interferometers to temperature 
not excited in this case. The temperature sensitivity for the LC coated tapered interferometer with a waist diameter of $13 \mu \mathrm{m}$ is $0.08 \mathrm{~nm} /{ }^{\circ} \mathrm{C}$. The sensitivity can be further increased by lowering the taper diameter. It should also be noted that the sensitivity presented in this paper is for the interferometer coated with the $1550 \mathrm{LC}$ material whose birefringence of 0.06 which is relatively low compared to other LC materials. Hence the sensitivity can be further increased by using low index LC materials with higher birefringence.

\subsection{Response of the interferometer covered with a high index LC layer}

To study the impact of high index LC materials on the tapered PCF interferometers, the $19 \mu \mathrm{m}$ waist diameter interferometer was coated with a layer of 5CB LC material. In the case of $5 \mathrm{CB}$ the clearing point temperature is $33{ }^{\circ} \mathrm{C}$. Hence the temperature of the interferometer is varied from $10{ }^{\circ} \mathrm{C}$ to $30{ }^{\circ} \mathrm{C}$ only. The measured spectra of the interferometer coated with $5 \mathrm{CB}$ at $10{ }^{\circ} \mathrm{C}$ and $30{ }^{\circ} \mathrm{C}$ are shown in Fig 9. It can be seen from the figure that no shift in the spectrum occurred with a temperature change. Also the extinction ratio of the spectrum is reduced further. This is because the higher refractive index of the $5 \mathrm{CB}$ causes the higher order modes to radiate away and therefore they do not take part in the interference. Hence high index LC materials are not suitable for temperature control of the PCF interferometer spectrum.

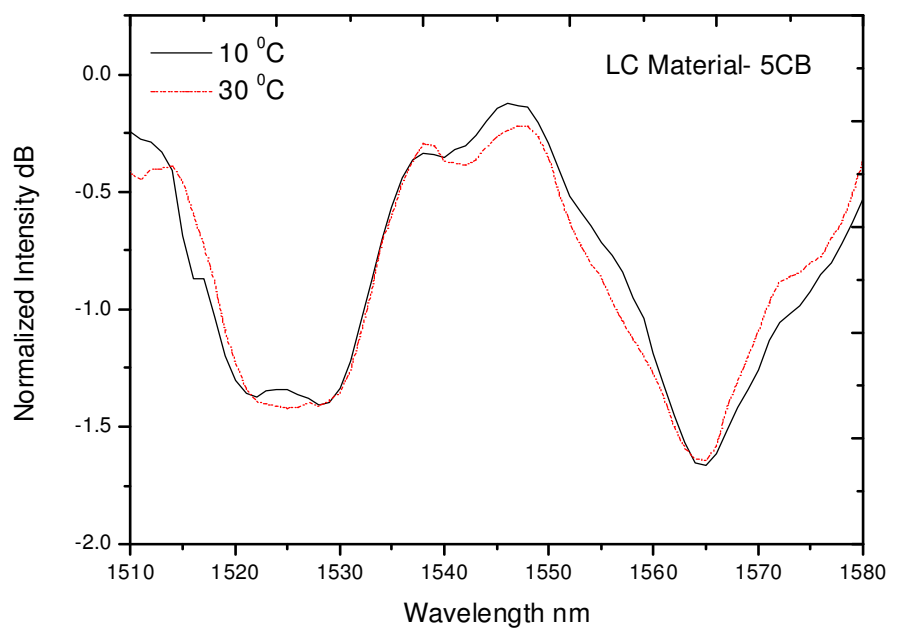

Figure 9. Spectral response of the tapered interferometer coated with 5CB LC material at two different temperatures

Thus in summary we have shown that LC materials with a low refractive index can be used for the purpose of controlling tapered PCF interferometers and that an interferometer with a smaller waist diameter shows higher sensitivity to the presence of the LC material. It should be noted that realizing such interferometers in practice may be difficult because of the lack of LC materials with average refractive indices below that of silica and also with high birefringence. One possible solution to overcome this problem is the use of commercially available high index LC materials together with tapers made from high index PCFs [18]. Further studies on the fabrication of tapered devices from high index soft glass PCFs are underway. Further studies are also underway to evaluate the response of the LC coated interferometer with an applied electric field to demonstrate electronic tunability of the PCF interferometers.

\section{Conclusion}

An experimental analysis and characterization of a liquid crystal coated tapered photonic crystal fiber interferometer was presented in this paper. Temperature dependence studies of tapered interferometers fabricated from different types of the photonic crystal fibers were 
carried out to select an interferometer to be coated with the liquid crystal material. It was found that interferometers fabricated from LMA-10 fiber were the most suitable for LC applications due to their very low intrinsic temperature dependence. Temperature tuning of the spectral response of the fabricated LC coated tapered interferometer with different waist diameters was investigated. It was also concluded that LC material with a low average refractive index should be used with tapered PCF interferometers. From the results it was shown that the temperature sensitivity of a LC coated tapered PCF interferometer depends on the waist thickness of the tapered region of the interferometer and that sensitivity is higher for interferometer with a lower waist diameter.

\section{Acknowledgements}

The authors would like to thank Prof. R. Dabrowski and Dr. E. N. Kruszelnicki of Military University of Technology, Warsaw, Poland for supplying the low index LC Material. The authors are grateful to Prof. T. R Wolinski and Dr. S Ertman of the Warsaw University of Technology, Poland for useful discussions on the low index LC materials.

This work is supported by the National Academy for Integration of Research, Teaching and Learning through the NAIRTL Grants Initiative 2009.

\section{References}

[1] Villatoro, J., Finazzi, V., Badenes, G., and Pruneri, V., "Highly sensitive sensors based on photonic crystal fiber modal interferometers," Journal of Sensors, 747803, 2009.

[2] Dong, X. and Tam, H., Y., "Temperature-insensitive strain sensor with polarizationmaintaining photonic crystal fiber based Sagnac interferometer," Applied Physics Letters, 91, 091109, 2007.

[3] Ju, J., Wang, Z., Jin, W., and Demokan, M, S., "Temperature sensitivity of a two-mode photonic crystal fiber interferometric sensor," IEEE Photo. Tech. Lett., Vol. 20, pp. 21682170, 2006.

[4] Rao, Y, J., Zhu, T., Yang, X, C., and Duan, D, W., "In-line fiber optic etalon formed by hollow-core photonic crystal fiber," Opt. Lett., Vol. 32, pp. 2662-2664, 2007.

[5] Shi, Q., Lv, F., Wang, Z., Jin L., Hu, J, J., Liu, Z., Kai, G., and Dong, X., "Environmentally stable Fabry-Perot type strain sensor based on hollow-core photonic bandgap fiber," IEEE Photo. Tech. Lett., Vol. 20, pp. 237-239, 2008.

[6] Frazao, O., Santos, J, L., Araujo, F, M., and Ferreira, L, A., "Optical sensing with photonic crystal fiber," Laser \& Photon. Rev., 2, pp. 449-459, 2008.

[7] Jha, R., Villatoro, J., Finazzi, V., Badenes, G., and Pruneri, V., "Refractometry based on a photonic crystal fiber interferometer," Opt. Lett., 34, pp. 617-619, 2009.

[8] Villatoro, J., Finazzi, V., Minkovich, V, P., Pruneri, V., and Badenes, G., "Temperatureinsensitive photonic crystal fiber interferometer for absolute strain sensing," Applied Physics Letters, 91, 091109, 2007.

[9] Minkovich, V, P., Villatoro, J., Hernandez, D, M., Calixto, S., Sotsky, A, B., and Sotskaya, L, I., "Holey fiber tapers with resonance transmission for high-resolution refractive index sensing", Optics Express, 13(19), pp. 7609-7614, 2005.

[10] Lcroix, S., Gonthier, F., Black, R. J., Bures, J., "Tapred-fiber interferometric wavelength response: the achromatic,” Opt. Lett., 13, pp. 395-397, 1988.

[11] Arregui, F, J., Matias, I, R., Lopez-Amo, M., "Optical fiber strain gauge based on a tapered single-mode fiber", Sensors and Actuators, 79, pp. 90-96, 2000.

[12] Lu, P., Men, L., Sooley., Chen, Q.,"Tapered fiber Mach-Zehnder interferometer for simultaneous measurement of refractive index and temperature", Applied Physics Letters, 94, 131110, 2009.

[13] Demus D., Gooby J., Gray G W., Spiess H W., Vill V., "Physical properties of liquid crystals", Wiley-VCH, Toronto, ON, Canada, 1999.

[14] Li, J., Gauza, S., and Wu, S, T., "Temperature effect on liquid crystal refractive indices", Journal of Applied Physics, 96(1), pp. 19-24, 2004. 
[15] Xiao, L., Demokan, M, S., Jin, W., Wang, Y., and Zhao, C, L., "Fusion splicing photonic crystal fibers and conventional single-mode fibers: microhole collapse effect," J. Lightwave Tech., Vol. 25, pp. 3563-3574, 2007.

[16] Magi, E, C., Steinvurzel, P., and Eggleton, B, J., “Tapered photonic crystal fibers”, Optics Express, 13(5), 776-784, 2004.

[17] Wolinski, T, R., Szaniawska, K., Ertman, S., Lesiak, P., Domanski, A, W., Dabrowski, R., Kruszelnicki, E, N., and Wojcik, J., "Influence of temperature and electrical fields on propagation properties of photonic liquid-crystal fibres", Meas. Sci. Technol., 17, pp. 985991, 2006.

[18] Ertman, S., Wolinski, T, R., Pysz, D., Buczynski, R., Kruszelnicki, E, N., and Dabrowski, R., "Low-loss propagation and continuously tunable birefringence in high-index photonic crystal fibers filled with nematic liquid crystals", Optics Express, 17(21), pp. 19298-19310, 2009. 